\title{
Understanding the Diverse Needs of Subtitle Users in a Rapidly Evolving Media Landscape
}

\author{
By Mike Armstrong, Andy Brown, Michael Crabb, Chris J. Hughes, Rhianne Jones, and James Sandford
}

\begin{abstract}
Audiences are increasingly using services, such as video on demand and the Web, to watch television programs. Broadcasters need to make subtitles available across all these new platforms. These platforms also create new design opportunities for subtitles along with the ability to customize them to an individual's needs. To explore these new opportunities for subtitles, we have begun the process of reviewing the guidance for subtitles on television and evaluating the original user research. We have found that existing guidelines have been shaped by a mixture of technical constraints, industry practice, and user research, constrained by existing technical standards. This paper provides an overview of the subtitle research at BBC RED over the past two years. Our research is revealing significant diversity in the needs and preferences of frequent subtitle users, and points to the need for personalization in the way subtitles are displayed. We are developing a new approach to the authoring and display of subtitles that can respond to the user requirements by adjusting the subtitle layout on the client device.
\end{abstract}

\section{Keywords}

Closed captions, subtitles accessibility, usability, UX, television, web, VoD, video, personalization, responsive, diversity

\section{Introduction}

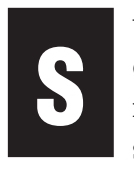
ubtitles $^{i}$ as an access service (also known as closed captions) were first broadcast on television in the U.K. over 35 years ago, using the Teletext system. Subtitles are now an integral part of the television service provided on all BBC programs in the U.K. via digital television services and are used by

Digital Object Identifier 10.5594/fMI.2016.2614919

Date of publication: 16 December 2016

iThis paper uses the U.K. nomenclature of "subtitles" rather than "closed captions," as it was originally written for a European audience. around $10 \%$ of the viewing audience every day. However, most of the research on subtitles was conducted using the Teletext system or other legacy formats with similar constraints. This has meant that progress in subtitle research has been quite conservative in its approach and has failed to address the challenges being posed by the way television content is now being watched on computers, tablets, and mobile phones. These new devices offer a very different experience from that of watching analog television 30 years ago. Furthermore, recent research on same-language subtitles has been diverted by academics whose expertise is in translation and who focus on promoting the role of the subtitler, rather than the experience of the audience. Our research has attempted to address issues with previous work and pay careful attention to the audience's experience of subtitles. We have begun to build a new model of the experience of watching television with subtitles and are finding some considerable variation in the wants and needs of different subtitle users, depending on their sensory and cognitive abilities, and the way in which they use the subtitles. To best meet these diverse needs and the new media landscape, we have proposed a new approach to subtitles that we call responsive subtitles.

\section{Background}

Teletext subtitles first appeared on BBC Television in the U.K. in 1979 and live subtitles were first broadcast in 1984. In 2008, the BBC achieved $100 \%$ subtitling for all of its main channels and is now providing subtitles for $98 \%$ of the programs on its video-on-demand service iPlayer.

A large proportion of the U.K. television audience relies on subtitles. The BBC's audience research team has run two audience surveys for us over the past two years. Each used a representative sample of around 5000 participants, who were questioned on that day's 
viewing. The responses indicate that about $10 \%$ of the audience use subtitles on any one day and around $6 \%$ use them for most of their viewing. This equates to an audience of around 4.5 million people in the U.K., of which over 2.5 million use them most or all of the time. Importantly, not all subtitle users have hearing difficulties, some are watching with the sound turned off and others use them to support their comprehension of the program, while around a quarter of people with hearing difficulties watch television without subtitles. ${ }^{1}$

\section{Research and Regulation}

The original guidelines for subtitling in the U.K. were informed by research carried out in the late 1970s by a team based at Southampton University on behalf of the Independent Broadcasting Authority. This pioneering work was difficult because of the nature of available television equipment at the time, and subtitled television was a novel experience for the participants. ${ }^{2,3}$ The guidelines were published in 1982 and contained extensive guidance on how scripts should be edited to create subtitle blocks. ${ }^{4}$

The Centre for Deaf Studies in Bristol reviewed the research on television subtitling in a report for the BBC and Independent Television Commission (ITC) in 1992. The report raised concerns about the existing research and the lack of follow-up work in several areas, including the issue of whether subtitles should be edited or presented verbatim. ${ }^{5}$ In 1996, the ITC commissioned research on viewers' preference for block subtitles or scrolling subtitles for news coverage. The report recorded no strong preference for block or scrolling subtitles but argued for edited subtitles, despite a majority of respondents preferring verbatim subtitles. It also highlighted problems with subtitles being delayed and obscuring other information. ${ }^{6}$ The ITC published new guidelines in 1997, which were updated in 1999 to include the new digital television services and the move to Digital Video Broadcasting (DVB) subtitles. However, apart from specifying the Tiresias font, the approach replicated the existing Teletext delivery. ${ }^{7}$

Also in 1999, a book was published that attempted "to establish the common ground between intra- and inter-lingual subtitling." Based on research at the Centre for Deaf Studies, the book deliberately conflated subtitles for access services with subtitling for translation. ${ }^{8}$ It marks the start of the field of audiovisual translation where arts academics have focused on the subtitler and have been dismissive of user preferences, even framing them as "political"9 or dismissing them as "a huge mistake."10

Since Ofcom took over from the ITC, they commissioned research on subtitling speed ${ }^{11}$ with a view to revising the ITC guidelines, which they published following a public consultation in 2006. These new guidelines are considerably shorter than the ITC document, removing the guidance on editing and formatting of subtitles. However, they still contain some legacy issues inherited from Teletext. ${ }^{12}$ Since 2013, Ofcom has been focusing on a program of work measuring the quality of live subtitling. ${ }^{13}$

\section{The Changing Media Landscape}

In 2009, BBC Online published a set of guidelines outlining the subtitling requirements for $\mathrm{AV}$ content presented on the bbc.co.uk website. ${ }^{14}$ These were based on existing television subtitle guidelines and existing research. ${ }^{5,6,15}$ We have been reviewing the validity of this guidance for the new viewing platforms and viewing contexts. With television programs available on computers and portable devices, the context for television viewing is a more individual experience and increasingly portable. In fact, by the end of 2013, viewing of the BBC's iPlayer on tablets overtook viewing on computers. ${ }^{16}$

As we reviewed the BBC Online guidelines, we also found problems with the subtitling guidelines for television and the persistence of legacy constraints. The Teletext display grid of 24 rows by 40 columns was designed for CRT displays with interlaced scanning (Fig. 1). The font was fixed-width and a double-height font was used for subtitles. This layout has been used to conduct most research over the past 30 years. In the U.K., the format for subtitle delivery to the home has moved on. Since October 2012, most U.K. broadcasters deliver subtitles in DVB format and iPlayer uses the Timed Text Markup Language (TTML) format.

There is pressure from the audience (and regulators in some countries) to provide the ability to change the size of the subtitles, and the demand is likely to increase as more people use a variety of screen sizes. The current approach, whereby each subtitle is authored and displayed as a fixed block of text, restricts the ability to personalize the subtitle display. Increasing the font size will result in more of the video image being

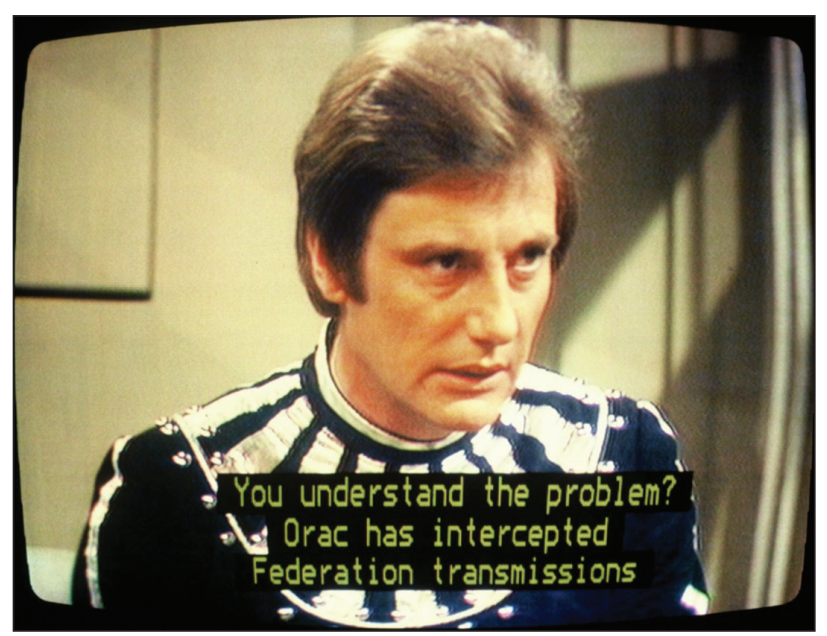

FIGURE 1. Teletext subtitles on a CRT TV. 
obscured, the subtitle exceeding the width of the screen, or both.

\section{New Challenges for Broadcasters}

This changing media landscape is also creating new challenges for the provision of subtitles. The levels of subtitling that have been achieved on U.K. broadcast television are not currently being matched by the provision of subtitles via video-on-demand services. The BBC iPlayer service provides subtitles on $98 \%$ of the content available, and the 2014 Authority for Television On Demand (ATVOD) services report shows that the public service broadcasters are making the most significant progress. However, in other cases, the level of provision is patchy, with nearly $15 \%$ of providers offering no subtitles at all via their websites. ${ }^{17}$ The campaign group Action on Hearing Loss has launched a campaign to improve subtitling provision for on-demand services. ${ }^{18}$ However, in the case of video clips on websites, the provision of subtitles by all broadcasters remains largely nonexistent, a situation we are actively addressing with our research work.

The issue of the quality of live subtitles continues to be a challenging problem. Action on Hearing Loss, drawing on feedback from its members, highlights the problems that subtitle users face when watching television. The most frequently reported issue was the delay between speech and the subtitles, closely followed by accuracy. The lack of subtitles or intermittent subtitles was also reported at a similar rate with other issues some way behind, such as subtitles covering faces or subtitles being too slow or too fast. ${ }^{19}$ Recent work by the BBC and its subtitling partners has been making significant improvements in live subtitle quality, particularly with live news bulletins, and further work is ongoing. Our work at BBC R\&D aims to support these improvements by understanding the needs of the audience and helping understand where improvements can have most benefit.

\section{Our Work}

Our aim, as a research team at BBC R\&D, is to provide the BBC with new knowledge about the use and experience of subtitles by our audiences and work out how best to develop subtitling for the new media landscape. We have been building a body of data on the quantity and quality of subtitling, both live and prerecorded, and we are now starting to create prototypes, which demonstrate a fresh approach to sourcing and providing subtitles. Our work in BBC R\&D is guided by the approach in the BBC's Diversity Strategy, which includes the objective to, "Build in accessibility from the start when developing new products and services, and ensure sustainable and on-going accessibility." 20 We are aiming to build a better understanding of the issues that affect the subtitle viewing experience, from the availability of subtitles across devices and platforms to the aspects of quality, the impact of the viewing device, and subtitles in a shared viewing experience.

Our work breaks down into three main areas: developing affordable and sustainable ways of increasing the availability of subtitles across all platforms, carrying out user research to understand the quality of the current user experience of subtitles for the audience, and research aimed at utilizing the characteristics of new platforms to improve the experience.

\section{Increasing Subtitle Availability}

The most important issue for the audience is the availability of subtitles. While the BBC aims to subtitle all of its programs and is also close to achieving that with its video-on-demand service iPlayer, subtitles are not currently available for the majority of clips on the BBC's Web pages. It would be too expensive to manually subtitle all of this content, but many of these clips contain content that has been broadcast as part of a television program and so have already been subtitled. We have been investigating ways in which we could locate and reuse broadcast subtitles. To demonstrate this, we have developed a prototype system, currently aimed at the BBC News website, which can determine when a video clip was broadcast and retrieve the subtitles from our off-air recordings.

Our system works by searching an archive of off-air recordings to match the video clip to identify the time at which it was broadcast and extracting the subtitles for this time segment. The speed of the search is improved by using the data from the Web page to reduce the number of programs that need to be searched. The audio signal is used to perform the comparison, because it relates directly to the words in the subtitles. Our system employs an audio fingerprinting algorithm to represent the broadcast content and Web clips to speed up the search while providing sufficient temporal accuracy.

A snapshot was taken of the BBC News website at one point in time to evaluate the proportion of clips that could be subtitled. The snapshot contained 346 video clips. Of these, $40 \%$ could be found automatically on a first search, as these clips had not been edited. When the search was extended to look for edited clips, it found matches for a further 20\%. Among the $40 \%$ of clips for which no match could be found, the most common causes for these failures were identified as the clip not having been broadcast, too heavily edited, or contained no audio.

An analysis of the results across the different subsections of the BBC News website found that different sections typically have different editorial requirements, resulting in differing levels of success, 


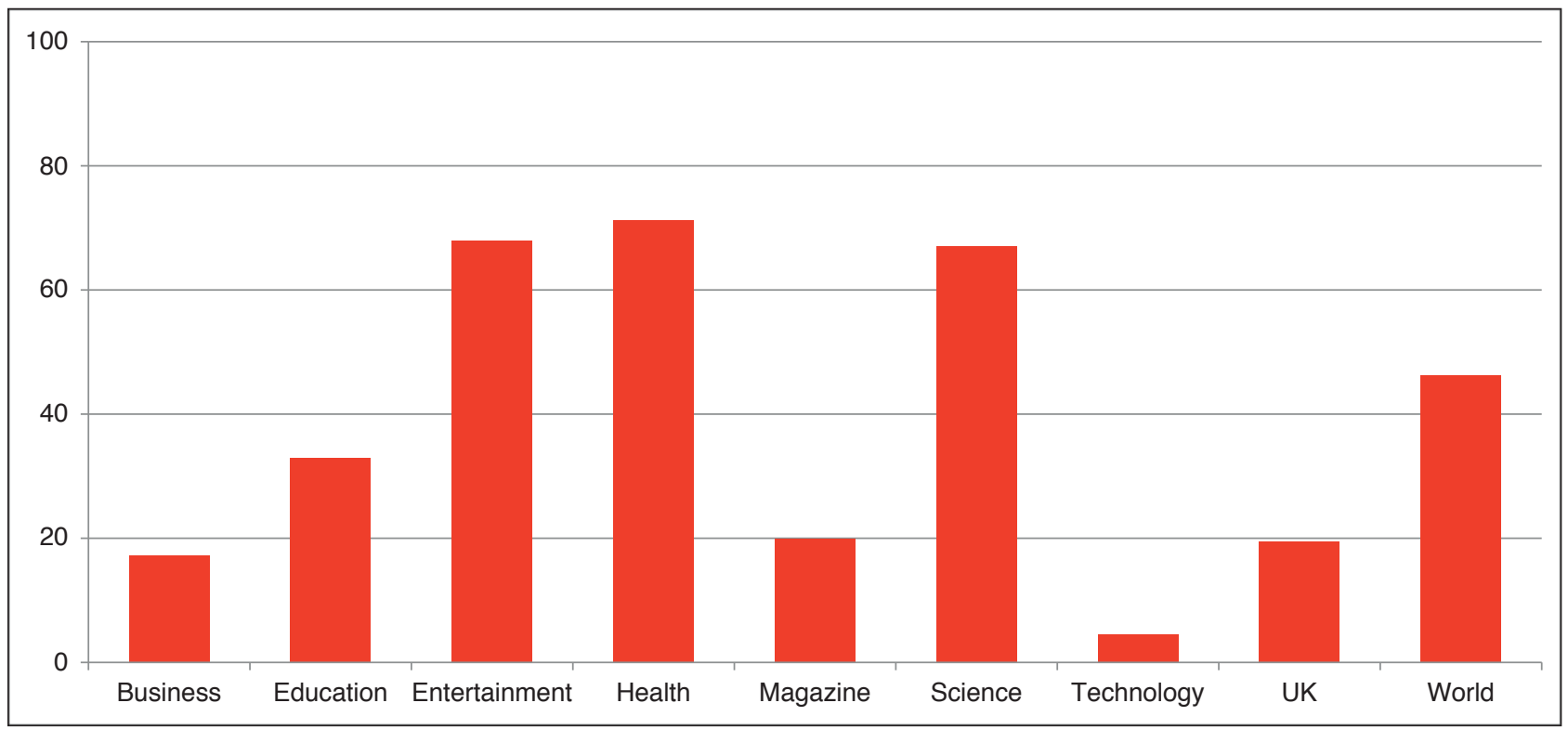

FIGURE 2. Success rate across subsections (\%).

as shown in Fig. 2. The highest success rate was found with "Entertainment," "Health," and "Science," where clips were generally taken directly from broadcast, while the "Technology" section provided the lowest success rate. This section contains a high proportion of content that has been made specifically for the Web. While our approach cannot provide subtitles for all clips on the BBC News website, our results show the considerable advances that could be made in subtitling broadcaster's websites in the future. This work was presented as a full paper at National Association of Broadcasters in $2015,{ }^{21}$ and a patent application covers this technique. ${ }^{\text {ii }}$

\section{Understanding the Experience of Audience}

Our approach to user research was built on the well-established engineering approach to measuring audio and video quality and was combined with techniques for measuring user experience from the fields of human-computer interaction (HCI) and accessibility research. This work is aided greatly by working with university partners, particularly the University of Dundee, that bring with them a wealth of experience of $\mathrm{HCI}$ and accessibility along with the experience of the issues of representing these user groups. ${ }^{22}$

As we reviewed the published user literature on subtitles, some worrying patterns emerged. We have identified a number of common problems in previous research that misrepresent the experience of regular subtitle users. These include the following:

- Test material shown without sound.

- Test material that excludes lipreading, such as cartoons, animation, and dubbed content.

\footnotetext{
${ }^{i i}$ Since this paper was originally written, this technique has been extended to recover subtitles for large numbers of clips from prerecorded programs without the need for human intervention. ${ }^{31}$
}

- The use of film content rather than content made for television.

- Tests carried out with convenience samples of participants who are not regular subtitle users and even the use of student subtitlers.

- Eye-gaze measurements made with the participant very close to a computer monitor and using a chin rest to constrain head movement, resulting in excessive eye movements.

Unfortunately while many research teams clearly signal the limitations of their work, some do not, and this problem is compounded when the limitations are not mentioned when the work is referenced. In addition, the research carried out on subtitles for translation is often referenced inappropriately in papers on samelanguage subtitles, and this is a particular problem in the field of audiovisual translation. The two types of subtitles have very different perceptual experiences. With translation subtitles, the audio and lip movements bear little relationship to the text so the viewer is simply reading, whereas with same language subtitles, the text acts in concert with hearing and lip-reading to enhance the viewer's understanding of the program.

These differences have been made clear to us by many of the participants in our user research, all of whom are frequent users of same-language subtitles. Also our survey data showed that around $90 \%$ of people using subtitles also have the sound on. In this condition, the sound, lip movements, and subtitles work together to provide the viewing experience; in effect, the subtitles are priming the auditory system. As one participant put it:

“...so I'm reading and hearing but the hearing only works if I'm reading-putting two and two together." 
For this user and many others, subtitles provide perceptual priming, enabling the person to recognize the words when they hear them spoken. This was reflected by another participant, who used subtitles so that he could watch with normal sound levels:

"I turn the volume up and people start complaining so [I use subtitles] to help hear.”

For others, the subtitles act as a failsafe system, only glanced at when the hearing and/or lip reading doesn't provide the information:

"My hearing sometimes is not that great [so I] use [subtitles] to double check."

"So I'm watching, I'm lip-reading, and I'm reading the words to check."

Because of the issues that we have found in previous research, we have been particularly careful to make our tests as realistic as possible for our participants, using a test lab that replicates a living room environment along with a normal television or computer as appropriate. We have used structured interviews alongside quantitative measures to draw out the participants' experiences of using subtitles in general and their views on our test examples. Where we have used eye-gaze tracking equipment, it is with a normal television at a normal viewing distance. We have also used realistic test material, carefully selected from our broadcast archive or created content that closely matches normal broadcasts. We use an external recruitment agency to provide test participants who represent the wide range of subtitle users. We recruited based on subtitle use rather than hearing ability, giving us insight into the full range of user experiences for subtitle users with differing accessibility needs.

\section{Delay and Accuracy}

Our first major piece of user research into the quality of live subtitles was commissioned in an attempt to quantify the relative impact of delay and accuracy on the perceived quality of subtitles. This work was published at IBC2013 and showed the big impact that delay has on the perceived quality of subtitles and highlighted the importance of sound on the viewer's experience. ${ }^{23}$ This work helped stimulate significant improvements to the subtitling of news programs in the U.K., and it is now commonplace to see subtitles being presented in sync with the newsreader on live television. This has been achieved by utilizing the same script data that are driving the presenter's autocue, thus also improving accuracy. In addition, many packages are now subtitled in advance. These techniques cannot address delay and accuracy in segments of news programs containing live interviews, and transitions between live and prescripted are especially difficult. However, it is a vast improvement, and there are now many short news bulletins broadcast in the U.K. that have fully accurate subtitles without any delay.

\section{Monitoring the Service}

A further key to understanding the quality of the experience of watching subtitles is being able to continuously monitor the service. In parallel with Ofcom's surveys of live subtitle quality based on short samples of programs selected by Ofcom every six months, ${ }^{13}$ we have developed a prototype subtitle monitoring system. This has enabled us to plot various parameters of the subtitles across all our main television services on a $24 / 7$ basis. ${ }^{24}$ While our system cannot measure delay and accuracy, it gives a continual measure of the presence or absence of subtitles, the subtitle word rate, the subtitle format, and the vertical position on the screen. This has provided baseline data on the service provided and helped identify a number of fault conditions. One of the issues that came to light was the issue of subtitle word rate, and this led to an important piece of research into the impact of word rate on subtitle enjoyment and perceived speed. ${ }^{25}$

\section{Dynamic Subtitles}

In addition to the move to displaying video in smaller formats as part of a Web page or on mobile devices, larger television sets are now becoming more common. When viewing subtitles on a larger screen, the angle between the main action and the subtitle increases. For this scenario, we have tested a different approach in which the subtitles are moved closer to the point of interest in the scene, rather than at the bottom, as shown in Fig. 3.

The video material for this test was a $1 \mathrm{~min} 50 \mathrm{sec}$ clip from the TV drama "Sherlock." This segment contained 34 subtitle blocks. The subtitles were positioned according to a number of factors: the character speaking the line, the background, and the position of the previous and subsequent subtitles. The subtitles were displayed as white text with a slim black outline. Participants watched the clip on a 47-in. television, and an eye-tracker, placed on a coffee table in front of the television, was used to record the gaze of participants as they viewed the clip.

Of the 26 participants, there was a clear division of opinion over the experience of dynamic subtitles: 5 of them did not like it, 8 were broadly positive about the experience, and 12 were very keen on having them. Interestingly, the three participants who expressed the greatest dislike of dynamic subtitles all said that they did not rely on subtitles but used them to double check on

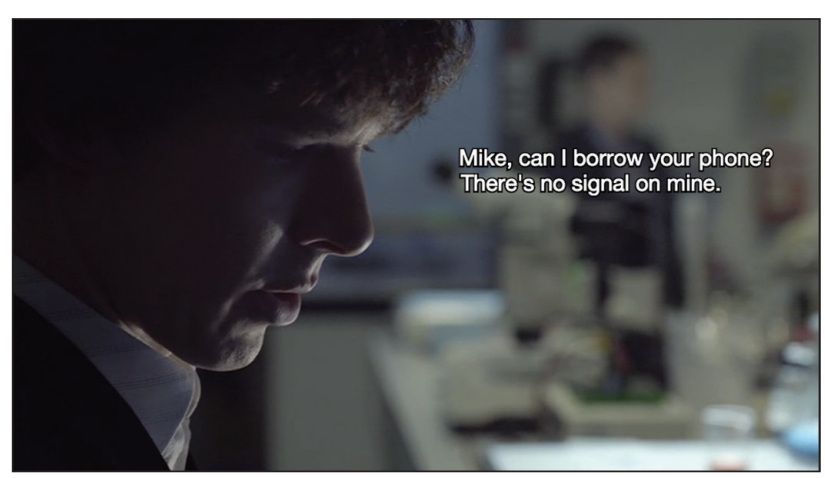

FIGURE 3. Example of dynamic subtitles. 
words they did not catch. By contrast, those who were very keen on dynamic subtitles said that they helped them see the little social cues and body language that they often miss with traditional subtitles and helped greatly with identifying who was speaking and make more sense of conversations. One even commented that the experience of seeing dynamic subtitle would spoil the experience of normal subtitles for them now. The eye tracking data confirmed that the gaze patterns of participants were significantly closer to the eye-gaze patterns for viewing without subtitles than normal subtitles and confirmed the results from an earlier set of tests. ${ }^{26}$

\section{Options for Subtitling on Web Pages}

New platforms for video content like video clips on Web pages or on mobile devices bring both challenges and opportunities. Challenges come about because of the smaller size and resolution of the video display, while opportunities arise because of the increased processing power in the client. Our initial user tests have looked at the user experience of viewing subtitles in a video embedded in a Web page.

\section{Subtitle Positioning on a Web Video}

One of the key differences between a video displayed on a television and a video clip on a Web page is that the video utilizes only part of the display area. This introduces the possibility of using part of the Web page outside of the video area to display subtitles. One suitable approach might be to display the subtitles just below the video: however, we could find little prior work that explored this approach. We conducted user research to find out whether the user experience can be improved by changing the position of subtitles from within a video clip to below and whether this was affected by the size of the video on the page (see Fig. 4). It also looked at whether users perceived any value in the ability to control the position. The subtitles were displayed in a semitransparent gray box in both locations, giving a similar look and feel in both cases.

The user tests were run with 26 participants who all used subtitles daily to watch television with sound and regularly used the internet to consume news and current affairs content. The participants were introduced to the position control and shown four video clips in each of the formats in a balanced design. The user experience for each one was measured using 14 questions on a 5-point Likert scale. These questions were set in a framework of seven measures of user experience: aesthetics, attention, involvement, familiarity, perceived usefulness, perceived usability, and endurability. A semistructured interview was then taken to probe further into the user experience.

The scores for each of the seven factors were combined to give an overall score for each condition. Statistical tests showed no statistical significance for the differences between the four individual cases, but when the scores were combined across the dimensions of size and position, there was no significant effect for size, but there was a small but significant improvement in user experience with the subtitles placed below the video rather than overlaying. Note that this result for the small video on a Web page is different from the preference for large TV viewing above, underlining the need to adapt the subtitles to the viewing context. In the discussion on the control of the position of subtitles, participants commented that they might select different positions for different types of content. Overall, participants expressed a need and desire for a feature to override the position of subtitles to suite their personal needs according to the context and content. ${ }^{27}$

\section{Font Size}

In a further piece of work on the display of subtitles on a video on a Web page, we looked at the impact of

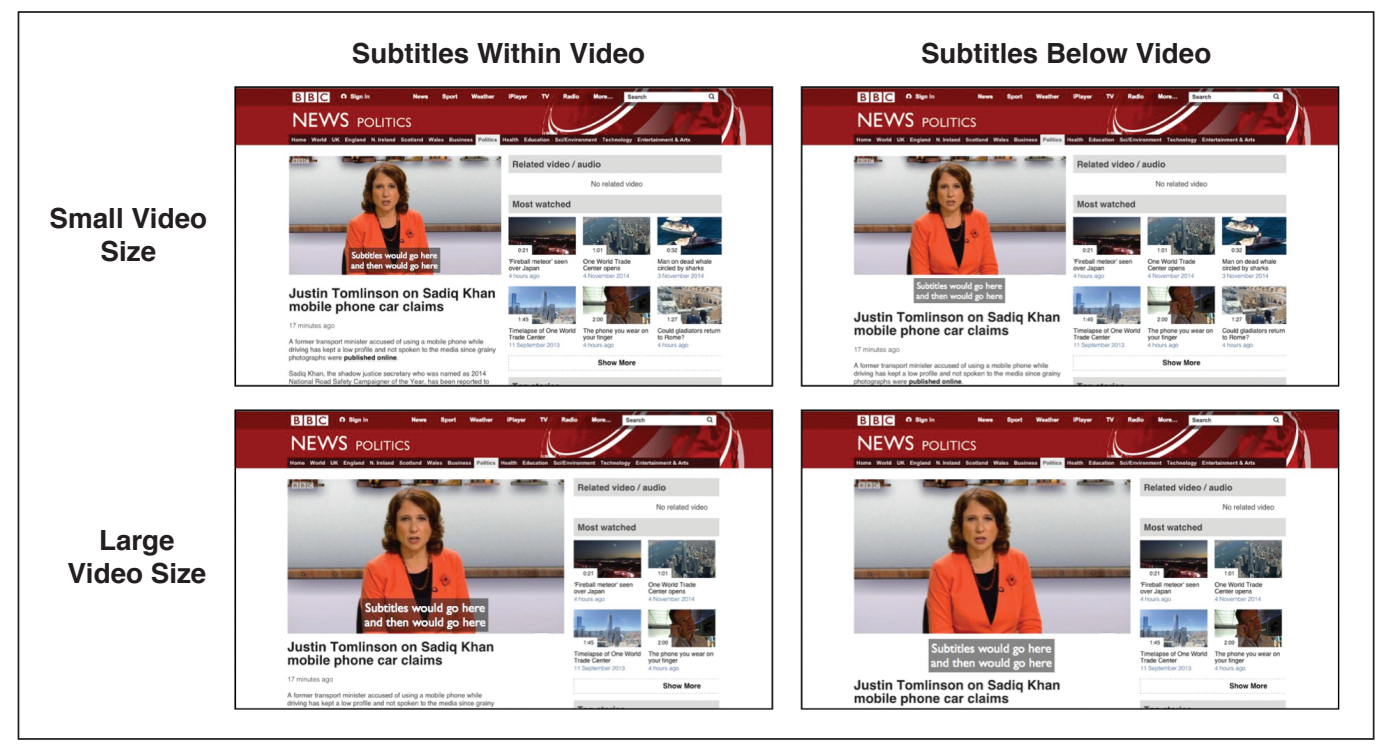

FIGURE 4. Layouts used for testing subtitle position on a video on a Web page. 
providing a control for changing the font size of the subtitles. The subtitles were formatted in the client in a responsive manner (see below), so the number of words displayed was changed to fit the space available while maintaining correct timing as the size of the font was adjusted. The participants viewed two video clips, the first from a magazine program and the second from a drama series.

The initial results showed that the size to which participants set the subtitles varied substantially (Fig. 5). Some preferred a smaller font as it allowed more words to be displayed at one time, as one participant put it, "I like it like this with more information. It makes more sense rather than splitting up into shorter sentences.” Others preferred having larger text: "Its great being able to make it bigger, it's almost like I can hear it better." In all of these recent pieces of research, we have found that subtitle users vary in their requirements from subtitles and that their needs vary according to the content they are viewing and the way in which they are viewing it. We have developed an approach that we think will enable the viewer to customize subtitles to best fit their needs and preferences, building on the wider object-based approach to broadcasting, which we are developing at BBC R\&D. ${ }^{28}$

\section{A New Approach-Responsive Subtitles}

The viewing of television content on computers and portable devices containing substantial levels of processing power opens up the possibility of providing personalized subtitles, tailored to the needs of the viewer and the constraints of the device. Furthermore, this fits well with the fact that viewing on these devices is more often a solo experience in contrast to TV viewing, which is often a social activity.

The current subtitling paradigm is that subtitles are created as a complete text block laid over the video content with the assumption that what the subtitler creates will be replicated in what the viewer sees. While the audience were all watching similar sized television sets, this one-size-fits-all approach was reasonable. However, as subtitles are distributed via many different

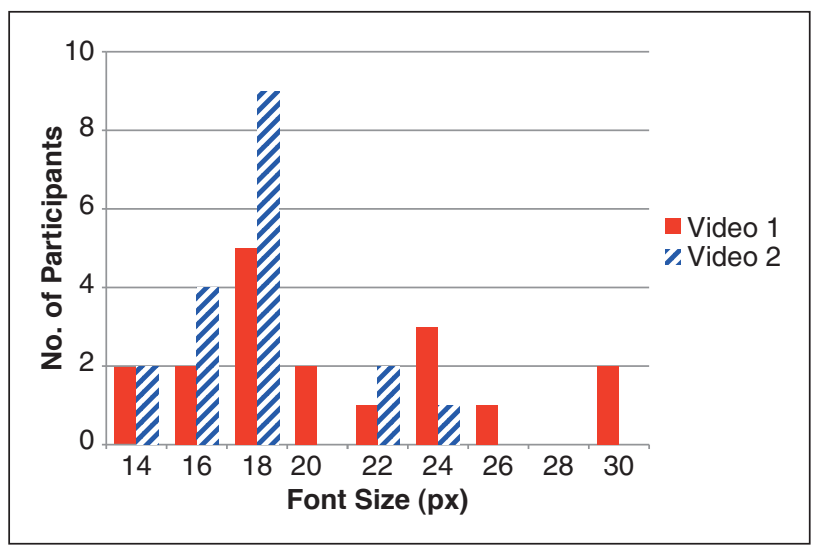

FIGURE 5. Distribution of font size choices. platforms, translated into different formats, and viewed on a wide range of devices, this approach breaks down. Also, as we have seen from our research, there are clear differences in the needs of subtitle users. A common complaint against the current TV systems is the inability to change the size of the subtitles. However, with current subtitle formats, if the size of the subtitles is increased, then they either obscure more of the video or spill off the edge of the screen, and sometimes both.

We have approached this problem by taking a lead from the world of Web design. We have based our work on the concept of responsive Web design, whereby the content is rendered into the available space using a fluid method, filling the space available on the display up to a maximum column width or line length. We call this technique responsive subtitles. ${ }^{29}$ In this approach, the content of the subtitle block is decided in the display device, using timings assigned to each word and rules that avoid orphaned words and uneven line lengths. The key advantage of this approach is that if the user changes the font or alters the size of the font, then the subtitle blocks are adjusted to fit within a well-defined display area. If a large font is used, then fewer words are displayed on screen, for a shorter time, and if a smaller font is chosen, then more text can be made available for longer time (Fig. 6).

This approach opens up further possibilities for personalization and enhanced display options. These can be under the control of the viewer, but broadcasters could also offer customization options. For example, a broadcaster could provide location data that place the subtitles close to the speaker for a drama series as proposed by Brown et al. ${ }^{26}$ and even render subtitles as speech bubbles but still retain the option of a conventional subtitling experience. Further user research will be needed to validate this concept, understand how users will exploit

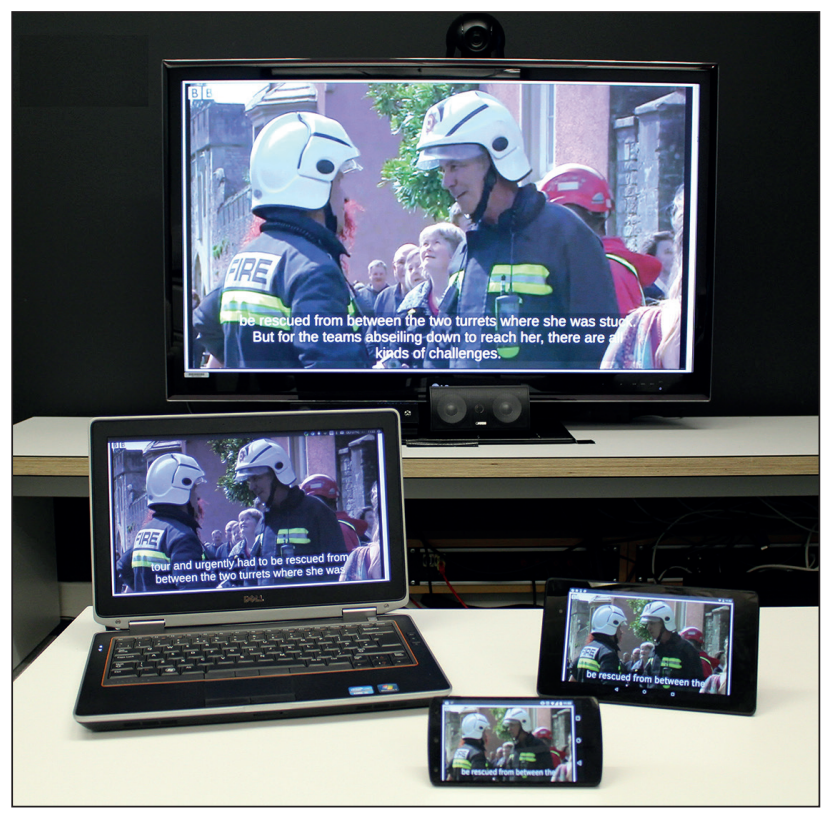

FIGURE 6. Responsive subtitles demo. 
it, and highlight where we can make improvements. This approach may also provide a solution for subtitling companies that face the problem of having to author different versions of subtitles for different markets. By restyling subtitles using supporting metadata, providers could make multiple versions more efficiently. ${ }^{30}$

\section{Conclusion}

Subtitles are a vital part of the viewing experience for a large proportion of the population in the U.K. Subtitle research and regulation have changed over time; however, because of shortcomings in some of the previous research and the conflation of same-language subtitles for accessibility with the separate subject of subtitles for translation, there are still major gaps in our knowledge. Therefore, a great deal more research is needed to fully understand the experience of viewing television with same-language subtitles and usefully update current guidance on subtitling. Our work in BBC R\&D is based in the scientific method and on an understanding of human perception and the accessibility context. We are discovering a significant diversity in the needs of different subtitle users and we are exploring ways of providing personalization for the audience.

Despite considerable changes in technology over the past 35 years, subtitling has been constrained by legacy standards, particularly Teletext, and has only recently started to move forward with new formats. This situation contrasts with the developments in television distribution, viewing habits, and viewing technology. New opportunities are opening up that can be used to further improve the experience of viewing subtitles by customizing subtitles to fit individual preferences and display capabilities.

To meet these needs, we have developed an approach that renders subtitles in a flexible manner. Building on responsive Web design, we therefore call responsive subtitling, and we have developed a prototype system that is controlled by style sheets, which thus offers a great deal of flexibility for the user, the subtitle provider, and the broadcaster. Our approach is one that could be used to meet the needs of different subtitle users who may be watching the same content across a wide range of devices. It also provides the option of personalization to meet the individual subtitle user's needs.

\section{Acknowledgments}

We would like to thank Gareth Ford-Williams from BBC Digital, Nigel Megitt from BBC Engineering, Simon Smith, and Richard Kurzik from BBC Television for their continued support and enthusiasm for this work. We would also like to credit Matthew Shotton, Matthew Brooks, and Samuel Bason with their contributions to this research. Many of the BBC R\&D publications referenced are also available as White Papers on the $\mathrm{R} \& \mathrm{D}$ website.

\section{References}

1. Royal National Institute for Deaf People (RNID), Annual Survey Report 2008, RNID, London, U.K. p. 12, 2008.

2. R. G. Baker and A. F. Newell, "Teletext Subtitles for the DeafProblems in Linguistics and Psychology," IBC 1980. 97-100, 1980.

3. A. F. Newell, "Teletext for the deaf," Electronics and Power, 28(3): 263-266, Mar. 1982.

4. R. Baker, A. Lambourne, and G. Rowston, Handbook for Television Subtitlers. Engineering Division, Independent Broadcasting Authority, London, U.K., 1982.

5. J. G. Kyle, Switched on: Deaf People's Views on Television Subtitling. University of Bristol, Centre for Deaf Studies, Bristol, U.K., 1992.

6. J. Sancho-Aldridge, Good News for Deaf People: Subtitling of National News, Programmes, Independent Television Commission, London, U.K., 1997.

7. ITC Guidance on Standards for Subtitling, Independent Television Commission, London, U.K., 1999.

8. Z. De Linde and N. Kay, The Semiotics of Subtitling, St. Jerome: Manchester, U.K., 1999.

9. P. Pablo Romero-Fresco, "More Haste Less Speed: Edited vs Verbatim Respeaking," Vigo Int. f Appl. Linguistics (VIAL), VI: 109-133, 2009.

10. J. Neves, "10 Fallacies about Subtitling for the d/Deaf and the Hard of Hearing," f Specialised Translation, (10):128-143, 2008.

11. "Subtitling-An Issue of Speed?" Ofcom, Jan. 2005, https://www.ofcom.org.uk/.

12. "Television Access Services Review," Ofcom, Annex 2, Guidelines on the Provision of Television Access Services, Sep. 2006.

13. "Measuring the Quality of Live Subtitling," Ofcom, Oct. 2013. 14. G. Ford-Williams, Online Subtitling Editorial Guidelines V1.1., BBC, 2009, bbc.co.uk.

15. S. Gregory and J. Sancho-Aldridge, Dial 888 Subtitling for Deaf Children. Independent Television Commission, 2013.

16. D. Taylor-Watt, "Christmas 2013 on BBC iPlayer," BBC Blog, Jan. 2014, www.bbc.co.uk/blogs/internet/entries/ a8e5725c-76ac-32ba-8f9f-af66393cba03.

17. ATVOD 2014, "Provision of Video on Demand Access Services-2014 Report," http:/www.atvod.co.uk/uploads/files/ Provision_of_Access_Services_2014_Report_FINAL.pdf.

18. Action on Hearing Loss, 2015, "Subtitle it! Whatever We Watch, However We Watch It," http://www.actiononhearingloss. org.uk/get-involved/campaign/access-to-television/subtitle-it.aspx. 19. L. Matthews, 2013, "Getting the Full Picture, Viewers' Experience of Television Subtitling. Action on Hearing Loss," http://www.actiononhearingloss.org.uk/supporting-you/policyresearch-and-influencing/research/getting-the-full-picture.aspx. 20. BBC, The BBC's Diversity Strategy 2011-15. p. 1, 2011.

21. C. J. Hughes and M. Armstrong, "Automatic Retrieval of Closed Captions for Web Clips from Broadcast TV Content," National Association of Broadcasters Conference, pp. 318-324. Apr. 2015. 22. A. Sears and V. L. Hanson, "Representing Users in Accessibility Research," CHI 2011: Conf. Proc. and Extended Abstracts, pp. 2235-2238. 2011.

23. M. Armstrong, "The Development of a Methodology to Evaluate the Perceived Quality of Live TV Subtitles," IBC2013 Conference, p. 11.1. Sep. 2013.

24. M. Armstrong, "Measurement of Subtitle Quality: An R\&D Perspective." in Subtitling: A Collective Approach, University of Nottingham, BBC R\&D White Paper WHP 255, Jul. 2013.

25. J. Sandford, "The Impact of Subtitle Display Rate on Enjoyment Under Normal Television Viewing Conditions," IBC2015 Conference, Sep. 2015.

26. A. Brown, R. Jones, M. Crabb, J. Sandford, M. Brooks, C. Jay, and M. Armstrong, "Dynamic Subtitles: The User Experience," ACM TVX2015 Conference, pp. 103-112, Jun. 2015. 
27. M. Crabb, R. Jones, A. Armstrong, and C. J. Hughes, "Online News Videos: The UX of Subtitle Position." ASSETS'15 Proc. 17th International ACM SIGACCESS Conference on Computers and Accessibility, pp. 215-222, Oct. 2015.

28. M. Armstrong, A. Brooks, M. Churnside, M. Evans, F. Melchior, and M. Shotton, "Object-based BroadcastingCuration, Responsiveness and User Experience," presented at IBC2014 Conference, Amsterdam, The Netherlands, Sep. 2014.

29. C. J. Hughes, M. Armstrong, R. Johnes, and M. Crabb, "Responsive Design for Personalised Subtitles," 12th Web for All Conference, Florence, Italy, Article No. 8, May 2015.

30. J. Birch, "Futures for Subtitling-A Manufacturer's View," Broadcast Film \& Video, 19(2):42, Feb. 2015.

31. M. Armstrong, "Automatic Recovery and Verification of Subtitles for Large Collections of Video Clips," presented at IBC2016 Conference, Amsterdam, The Netherlands, Sep. 2016.

\section{About the Authors}

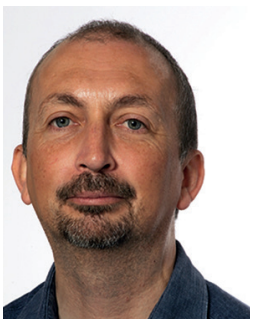

Mike Armstrong has led the BBC's research into access services over the past few years, triggering significant improvements in the BBC's subtitle services and having a major impact on the BBC's understanding of the audience's needs. He is now focusing on the challenges of object-based broadcasting and how metadata can be harnessed to automate production processes. Armstrong joined BBC R\&D in 1999, where he has developed a very broad expertise, covering a wide range of issues from access services, speech audibility, and video quality to visual perception, and is currently working on production techniques for object-based broadcasting. Previously, he worked in BBC Local Radio, where he gained a wide range of operational experience and pioneered the use of IT systems in broadcasting. He installed and ran the BBC's first ever server-based playout system at Radio Bristol.

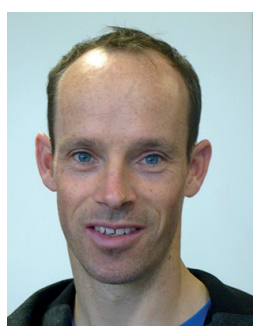

Andy Brown is a research scientist at BBC R\&D in Salford, U.K. He has a background in computer science, having earned a $\mathrm{PhD}$ and having worked as a Post-doctoral Research Associate at the University of Manchester, Manchester, U.K., in the fields of human-computer interaction and accessibility. $\mathrm{He}$ is currently working in the User Experience group, investigating how people interact with media, from production workflows to consumption by audience members, and from traditional media to new content experiences.

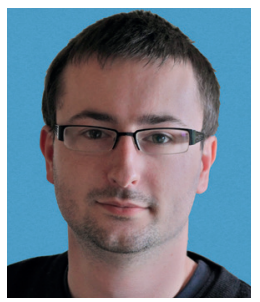

Michael Crabb is a lecturer in the School of Computer Science and Digital Media at Robert Gordon University, Aberdeen, U.K. He teaches in a variety of computingbased programs, with a focus on material related to Web development.
$\mathrm{He}$ is also an active researcher in the Robert Gordon User Experience Research Group, where his research interests surround the UX of accessible services. In the past, Crabb worked at the University of Dundee, Dundee, U.K., as part of the Engineering and Physical Sciences Research Council (EPSRC)-funded Social Inclusion in the Digital Economy project.

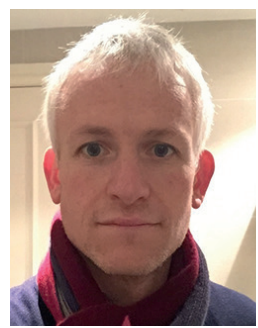

Chris Hughes is a lecturer in the School of Computer Science at Salford University, Salford, U.K. His research interests include data visualization and simulation, highperformance distributed computing, immersive media, and broadcast engineering. He has a particular interest in accessible services and is currently focused on developing new methods for providing accessibility services within an immersive context, such as virtual reality and $360^{\circ}$ video. Previously he worked for the UX group within BBC R\&D, where he was responsible for developing the concept of responsive subtitles and demonstrated several methods for automatically recovering and realigning subtitles for unknown clips.

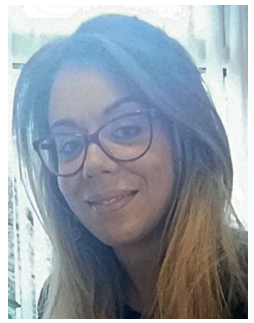

Rhianne Jones is a research scientist at BBC R\&D, where she co-runs the User Experience Lab. Jones is a social scientist and HCI practitioner, specializing in research into emerging technologies and trends. Jones current research focuses on inclusive design, new data-driven content experiences, and usable privacy.

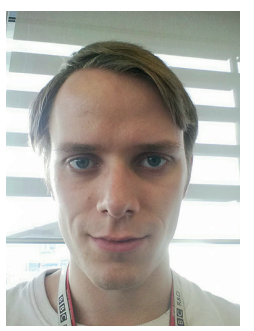

James Sandford is a research technologist in BBC's R\&D department. His current research focuses on methods of rendering ultra-high-definition content using streamable edit decision lists in IP broadcast infrastructures. Previously, Sandford has carried out research into methods of transporting and rendering subtitles in IP broadcast environments, user research into the preferred word rate of subtitles for the deaf and hard of hearing, and the methods of mixing and metering audio in IP broadcast environments. 